\title{
PRESENTATIONS OF FEIGIN-STOYANOVSKY'S TYPE SUBSPACES OF STANDARD MODULES FOR AFFINE LIE ALGEBRAS OF TYPE $C_{\ell}^{(1)}$
}

\author{
Goran TrupČEvić \\ University of Zagreb, Croatia
}

\begin{abstract}
Feigin-Stoyanovsky's type subspace $W(\Lambda)$ of a standard $\tilde{\mathfrak{g}}$-module $L(\Lambda)$ is a $\tilde{\mathfrak{g}}_{1}$-submodule of $L(\Lambda)$ generated by the highest-weight vector $v_{\Lambda}$, where $\tilde{\mathfrak{g}}_{1}$ is a certain commutative subalgebra of $\tilde{\mathfrak{g}}$. Based on the description of basis of $W(\Lambda)$ for $\tilde{\mathfrak{g}}$ of type $C_{\ell}^{(1)}$, we give a presentation of this subspace in terms of generators and relations

$$
W(\Lambda) \simeq U\left(\tilde{\mathfrak{g}}_{1}^{-}\right) / J
$$
\end{abstract}

\section{INTRODUCTION}

B. Feigin and A. Stoyanovsky introduced principal subspaces of standard modules for affine Lie algebras of type $A_{1}^{(1)}$ and $A_{2}^{(1)}$ in [12] where they have recovered Rogers-Ramanujan type identities by considering graded dimensions of these subspaces. An important part of their investigation was the knowledge of presentations of these subspaces in terms of generators and relations. Another type of principal subspaces, called Feigin-Stoyanovsky's type subspaces, was introduced by M. Primc who constructed bases of these subspaces in different cases $([24-26,18])$. These kind of subspaces were further studied by many authors $([28,15,10,11,1,13,14,2,4,5,17,30]$, etc.) and the knowledge of presentation presents an important question in this study $([6-9,27,23])$.

In our previous works we have described bases of Feigin-Stoyanovsky's type subspaces of standard modules for affine Lie algebras of type $C_{\ell}^{(1)}([3])$

2010 Mathematics Subject Classification. 17B67, 17B69, 05A19.

Key words and phrases. Affine Lie algebras, principal subspaces, generators and relations.

Partially supported by the Croatian Science Foundation grant 2634. 
and obtained from them basis for the whole standard modules ([3]). In this note we use the description of bases of a Feigin-Stoyanovsky's type subspaces to give presentations of these subspaces in terms of generators and relations.

\section{Feigin-Stoyanovsky's type subspaces}

Let $\mathfrak{g}$ be a complex simple Lie algebra of type $C_{\ell}$ with a Cartan subalgebra $\mathfrak{h}$ and a root decomposition $\mathfrak{g}=\mathfrak{h}+\sum \mathfrak{g}_{\alpha}$. Let

$$
R=\left\{ \pm \epsilon_{i} \pm \epsilon_{j} \mid 1 \leq i \leq j \leq \ell\right\} \backslash\{0\}
$$

be the corresponding root system realized in $\mathbb{R}^{\ell}$ with the canonical basis $\epsilon_{1}, \ldots, \epsilon_{\ell}$. Fix simple roots

$$
\alpha_{1}=\epsilon_{1}-\epsilon_{2}, \quad \ldots, \quad \alpha_{\ell-1}=\epsilon_{\ell-1}-\epsilon_{\ell}, \quad \alpha_{\ell}=2 \epsilon_{\ell}
$$

and let $\mathfrak{g}=\mathfrak{n}_{-}+\mathfrak{h}+\mathfrak{n}_{+}$be the corresponding triangular decomposition. Let $\theta=2 \alpha_{1}+\cdots+2 \alpha_{\ell-1}+\alpha_{\ell}=2 \epsilon_{1}$ be the maximal root and

$$
\omega_{r}=\epsilon_{1}+\cdots+\epsilon_{r}, \quad r=1, \ldots, \ell
$$

fundamental weights (cf. [16]). Fix root vectors $x_{\alpha} \in \mathfrak{g}_{\alpha}$. We identify $\mathfrak{h}$ and $\mathfrak{h}^{*}$ via the Killing form $\langle$,$\rangle normalized in such a way that \langle\theta, \theta\rangle=2$.

Let $\tilde{\mathfrak{g}}$ be the affine Lie algebra of type $C_{\ell}^{(1)}$ associated to $\mathfrak{g}$,

$$
\tilde{\mathfrak{g}}=\mathfrak{g} \otimes \mathbb{C}\left[t, t^{-1}\right]+\mathbb{C} c+\mathbb{C} d,
$$

with the canonical central element $c$ and the degree element $d$ (cf. [19]). Let

$$
\tilde{\mathfrak{g}}=\tilde{\mathfrak{n}}_{-}+\tilde{\mathfrak{h}}+\tilde{\mathfrak{n}}_{+},
$$

be a triangular decomposition of $\tilde{\mathfrak{g}}$, where $\tilde{\mathfrak{n}}_{-}=\mathfrak{n}_{-}+\mathfrak{g} \otimes t^{-1} \mathbb{C}\left[t^{-1}\right], \tilde{\mathfrak{h}}=$ $\mathfrak{h}+\mathbb{C} c+\mathbb{C} d, \tilde{\mathfrak{n}}_{+}=\mathfrak{n}_{+}+\mathfrak{g} \otimes t \mathbb{C}[t]$. Denote by $\Lambda_{0}, \ldots, \Lambda_{\ell}$ fundamental weights of $\tilde{\mathfrak{g}}$.

For $x \in \mathfrak{g}$ and $n \in \mathbb{Z}$ denote by $x(n)=x \otimes t^{n}$ and $x(z)=\sum_{n \in \mathbb{Z}} x(n) z^{-n-1}$, where $z$ is a formal variable.

Let $L(\Lambda)$ be a standard $\tilde{\mathfrak{g}}$-module with the highest weight

$$
\Lambda=k_{0} \Lambda_{0}+k_{1} \Lambda_{1}+\cdots+k_{\ell} \Lambda_{\ell},
$$

$k_{i} \in \mathbb{Z}_{+}$for $i=0, \ldots, \ell$, and fix a highest weight vector $v_{\Lambda}$. Denote by $k=\Lambda(c)$ the level of $\tilde{\mathfrak{g}}$-module $L(\Lambda), k=k_{0}+k_{1}+\cdots+k_{\ell}$.

Fix the minuscule weight $\omega=\omega_{\ell}=\epsilon_{1}+\cdots+\epsilon_{\ell} \in \mathfrak{h}^{*}$; then $\langle\omega, \alpha\rangle \in$ $\{-1,0,1\}$ for all $\alpha \in R$ and define the set of colors

$$
\Gamma=\{\alpha \in R \mid\langle\omega, \alpha\rangle=1\}=\left\{\epsilon_{i}+\epsilon_{j} \mid 1 \leq i \leq j \leq \ell\right\} .
$$

Write

$$
(i j)=\epsilon_{i}+\epsilon_{j} \in \Gamma \quad \text { and } \quad x_{i j}=x_{\epsilon_{i}+\epsilon_{j}} .
$$

This gives a $\mathbb{Z}$-gradation of $\tilde{\mathfrak{g}}$; let $\mathfrak{g}_{0}=\mathfrak{h}+\sum_{\langle\omega, \alpha\rangle=0} \mathfrak{g}_{\alpha}$, then

$$
\tilde{\mathfrak{g}}=\tilde{\mathfrak{g}}_{-1}+\tilde{\mathfrak{g}}_{0}+\tilde{\mathfrak{g}}_{1},
$$


where

$$
\tilde{\mathfrak{g}}_{0}=\mathfrak{g}_{0} \otimes \mathbb{C}\left[t, t^{-1}\right] \oplus \mathbb{C} c \oplus \mathbb{C} d, \quad \tilde{\mathfrak{g}}_{ \pm 1}=\sum_{\alpha \in \pm \Gamma} \mathfrak{g}_{\alpha} \otimes \mathbb{C}\left[t, t^{-1}\right] .
$$

The subalgebra $\tilde{\mathfrak{g}}_{1}$ is commutative, and $\mathfrak{g}_{0}$ acts on $\tilde{\mathfrak{g}}_{1}$ by adjoint action.

Feigin-Stoyanovsky's type subspace of $L(\Lambda)$ is a $\tilde{\mathfrak{g}}_{1}$-submodule of $L(\Lambda)$ generated by the highest-weight vector $v_{\Lambda}$,

$$
W(\Lambda)=U\left(\tilde{\mathfrak{g}}_{1}\right) \cdot v_{\Lambda}=U\left(\tilde{\mathfrak{g}}_{1}^{-}\right) \cdot v_{\Lambda} \subset L(\Lambda),
$$

where $\tilde{\mathfrak{g}}_{1}^{-}=\tilde{\mathfrak{g}}_{1} \cap \tilde{\mathfrak{n}}_{-}$.

We use an exponential notation to describe monomials $\mathbf{m} \in U\left(\tilde{\mathfrak{g}}_{1}^{-}\right)=$ $S\left(\tilde{\mathfrak{g}}_{1}^{-}\right)$:

$$
\mathbf{m}=\cdots x_{i^{\prime} j^{\prime}}(-n)^{b_{i^{\prime} j^{\prime}}} \cdots x_{i j}(-1)^{a_{i j}} .
$$

It will be clear from the context to which factors exponents $a_{i j}$ 's, $b_{i j}$ 's, $c_{i j}$ 's correspond to.

A monomial $\mathbf{m}$ is said to satisfy difference conditions for $W(\Lambda), D C$ for short, if for any $n \in \mathbb{N}$ and $i_{1} \leq \cdots \leq i_{t} \leq j_{t} \leq \ldots \leq j_{1} \leq i_{t+1} \leq \cdots \leq i_{s} \leq$ $j_{s} \leq \ldots \leq j_{t+1}$, the exponents of $x_{i j}(-n)$ 's and $x_{i j}(-n-1)$ 's in $\mathbf{m}$, denoted by $a_{i j}$ 's and $b_{i j}$ ', respectively, satisfy

$$
b_{i_{1} j_{1}}+\ldots+b_{i_{t} j_{t}}+a_{i_{t+1} j_{t+1}}+\ldots+a_{i_{s} j_{s}} \leq k .
$$

A monomial $\mathbf{m}$ satisfies initial conditions for $W(\Lambda), I C$ for short, if for every $i_{1} \leq \ldots \leq i_{t} \leq j_{t} \leq \ldots \leq j_{1}$,

$$
a_{i_{1} j_{1}}+\ldots+a_{i_{t} j_{t}} \leq k_{0}+k_{1}+\ldots+k_{j_{1}-1}
$$

where $a_{i j}$ 's denote exponents of $x_{i j}(-1)$ in $\mathbf{m}$.

THEOREM 2.1 ([3]). The set

$$
\left\{\mathbf{m} v_{\Lambda} \mid \mathbf{m} \text { satisfies } D C \text { and } I C \text { for } W(\Lambda)\right\}
$$

is a basis of $W(\Lambda)$.

\section{Presentation of Feigin-Stoyanovsky's type subspaces}

Difference conditions are consequences of the adjoint action of $\mathfrak{g}_{0}$ on the vertex-operator relation

$$
x_{\theta}(z)^{k+1}=0,
$$

or, equivalently, on a family of relations

$$
\sum_{\substack{n_{1}, \ldots, n_{k+1} \geq 1 \\ n_{1}+\cdots+n_{k+1}=N}} x_{11}\left(-n_{1}\right) \cdots x_{11}\left(-n_{k+1}\right)=0, \quad \text { for } N \geq k+1
$$

on $L(\Lambda)$ (cf. [3]; see also $[21,22,20]))$.

Root vectors of $\mathfrak{g}$ can be chosen so that the action of $\mathfrak{g}_{0}$ on $\mathfrak{g}_{1}$ is given by

$$
\left[x_{-\alpha_{i}}, x_{i j}\right]=x_{i+1, j}, \quad\left[x_{-\alpha_{j}}, x_{i j}\right]=x_{i, j+1}, \quad\left[x_{-\alpha_{i}}, x_{i i}\right]=2 x_{i, i+1}
$$


(cf. [16]). Then one easily sees that the adjoint action gives the following family of relations on $L(\Lambda)$ :

$$
\sum_{\substack{n_{1}+\cdots+n_{k+1}=N \\\left\{i_{1}, \ldots, i_{k+1}, j_{1}, \ldots, j_{k+1}\right\}=\left\{1^{m_{1}}, \ldots, \ell^{m_{\ell}}\right\}}} C_{\mathbf{i j}} x_{i_{1} j_{1}}\left(-n_{1}\right) \cdots x_{i_{k+1} j_{k+1}}\left(-n_{k+1}\right)=0,
$$

for some nonnegative integers $C_{\mathbf{i j}}$, where the sum runs over all such partitions $\mathbf{i}, \mathbf{j}$ of a multiset $\left\{1^{m_{1}}, \ldots, \ell^{m_{\ell}}\right\}, m_{1}+\cdots+m_{\ell}=2(k+1)$ (cf. [3]).

One obtains the difference conditions by finding minimal monomials of these relations, the so called leading terms of relations, whose multiples can be excluded from the spanning set. For this, a linear order on monomials is introduced. Define a linear order on the set of colors $\Gamma$ : $\left(i^{\prime} j^{\prime}\right)<(i j)$ if $i^{\prime}>i$ or $i^{\prime}=i, j^{\prime}>j$. On the set of variables $\tilde{\Gamma}^{-}=\left\{x_{\gamma}(n) \mid \gamma \in \Gamma, n \in \mathbb{Z}_{-}\right\}$define a linear order by $x_{\alpha}(n)<x_{\beta}\left(n^{\prime}\right)$ if $n<n^{\prime}$ or $n=n^{\prime}, \alpha<\beta$. For monomials, assume that factors descend from right to left, then use a lexicographic order (compare factors the greatest to the lowest one). Order $<$ is compatible with multiplication (see $[24,30])$ :

$$
\text { if } \mathbf{m}_{1}<\mathbf{m}_{2} \text { then } \mathbf{m m}_{1}<\mathbf{m m}_{2} \text {, for } \mathbf{m}, \mathbf{m}_{1}, \mathbf{m}_{2} \in U\left(\tilde{\mathfrak{g}}_{1}^{-}\right) \text {. }
$$

For initial conditions consider decompositions

$\Lambda=\Lambda^{(r)}+\Lambda_{(r)}, \quad \Lambda^{(r)}=k_{0} \Lambda_{0}+\cdots+k_{r-1} \Lambda_{r-1}, \quad \Lambda_{(r)}=k_{r} \Lambda_{r}+\cdots+k_{\ell} \Lambda_{\ell}$, for $1 \leq r \leq \ell$. By $v^{(r)}$ and $v_{(r)}$ denote highest weight vectors of the associated standard modules $L\left(\Lambda^{(r)}\right)$ and $L\left(\Lambda_{(r)}\right)$ of level $k^{(r)}=k_{0}+\cdots+k_{r-1}$ and $k_{(r)}=k_{r}+\cdots+k_{\ell}$, respectively. Then $L(\Lambda)$ can be embedded in a tensor product $L(\Lambda) \subset L\left(\Lambda^{(r)}\right) \otimes L\left(\Lambda_{(r)}\right)$. Since $x_{i j}(-1) v_{\Lambda_{r}}=0$ if and only if $j \leq r$ (cf. [3]), we have

$$
\mathbf{m}\left(v^{(r)} \otimes v_{(r)}\right)=\left(\mathbf{m} v^{(r)}\right) \otimes v_{(r)}
$$

for $\mathbf{m}=x_{i_{1} j_{1}}(-1) \cdots x_{i_{t} j_{t}}(-1)$ such that $j_{s} \leq r, 1 \leq s \leq t$. Hence, relations between such monomials corresponding to difference conditions for $W\left(\Lambda^{(r)}\right)$ automatically become relations in $L(\Lambda)$ (cf. [3]). This gives the following family of relations on $L(\Lambda)$ :

$$
\sum_{\left\{i_{1}, \ldots, i_{k+1}, j_{1}, \ldots, j_{k+1}\right\}=\left\{1^{m_{1}}, \ldots, r^{m_{r}}\right\}} C_{\mathbf{i j}} x_{i_{1} j_{1}}(-1) x_{i_{2} j_{2}}(-1) \cdots x_{i_{k}(r)+1} j_{k(r)+1}(-1)=0,
$$

for some nonnegative integers $C_{\mathbf{i j}}$, where the sum runs over all such partitions $\mathbf{i}, \mathbf{j}$ of a multiset $\left\{1^{m_{1}}, \ldots, r^{m_{r}}\right\}, m_{1}+\cdots+m_{r}=2\left(k^{(r)}+1\right)$.

Alternatively, for $r \geq 2$ let $\mathfrak{g}_{(r)} \subset \mathfrak{g}_{0}$ be the subalgebra generated by elements $x_{ \pm \alpha_{t}}, 1 \leq t<r$. Start from a relation

$$
x_{11}(-1)^{k^{(r)}+1}\left(v^{(r)} \otimes v_{(r)}\right)=\left(x_{11}(-1)^{k^{(r)}+1} v^{(r)}\right) \otimes v_{(r)}=0 .
$$


Now the adjoint action of $\mathfrak{g}_{(r)}$ on the above relation gives relations (3.3). For $r=1$, relations $(3.3)$ come down to only one relation

$$
x_{11}(-1)^{k_{0}+1}\left(v^{(1)} \otimes v_{(1)}\right)=0 .
$$

Recall that Feigin-Stoyanovsky's type subspace $W(\Lambda)$ is

$$
W(\Lambda)=U\left(\tilde{\mathfrak{g}}_{1}^{-}\right) \cdot v_{\Lambda}
$$

Since $\tilde{\mathfrak{g}}_{1}$ is commutative, universal enveloping algebra of $\tilde{\mathfrak{g}}_{1}^{-}$is isomorphic to a polynomial algebra $\mathbb{C}\left[\tilde{\Gamma}^{-}\right]$. Hence, there is a surjection

$$
f_{\Lambda}: \mathbb{C}\left[\tilde{\Gamma}^{-}\right] \rightarrow W(\Lambda), \quad f: \mathbf{m} \rightarrow \mathbf{m} \cdot v_{\Lambda} .
$$

We want to describe the kernel of this map, $\operatorname{ker} f_{\Lambda} \subset \mathbb{C}\left[\tilde{\Gamma}^{-}\right]$, so that

$$
W(\Lambda) \simeq \mathbb{C}\left[\tilde{\Gamma}^{-}\right] / \operatorname{ker} f_{\Lambda}
$$

as vector spaces.

Theorem 3.1. Let $J_{\Lambda} \subset \mathbb{C}\left[\tilde{\Gamma}^{-}\right]$be the ideal generated by the following sets

$$
\begin{gathered}
U\left(\mathfrak{g}_{0}\right) \cdot\left(\sum_{\begin{array}{c}
n_{1}, \ldots, n_{k+1} \geq 1 \\
n_{1}+\cdots+n_{k+1}=N
\end{array}} x_{11}\left(-n_{1}\right) \cdots x_{11}\left(-n_{k+1}\right)\right), \quad \text { for } N \geq k+1, \\
U\left(\mathfrak{g}_{(r)}\right) \cdot x_{11}(-1)^{k^{(r)}+1}, \quad \text { for } r=2, \ldots, \ell, \\
x_{11}(-1)^{k_{0}+1} .
\end{gathered}
$$

Then $\operatorname{ker} f_{\Lambda}=J_{\Lambda}$.

Proof. From (3.1), (3.4) and (3.5), see also (3.2) and (3.3), it follows that the generators of $J_{\Lambda}$ lie in the kernel of $f_{\Lambda}$. Hence $f_{\Lambda}$ can be factorized to a quotient map

$$
\overline{f_{\Lambda}}: \mathbb{C}\left[\tilde{\Gamma}^{-}\right] / J_{\Lambda} \rightarrow W(\Lambda) .
$$

This map is clearly a surjection, since $f_{\Lambda}$ is a surjection.

We can imitate the proof for the spanning set for $W(\Lambda)$ (cf. Proposition 2 and 4 in [3]) to reduce the spanning set for $\mathbb{C}\left[\tilde{\Gamma}^{-}\right] / J_{\Lambda}$. Consider the generators of $J_{\Lambda}$ and identify the minimal monomial inside each one; their multiples can be excluded from the spanning set. Like in [3], we get

$$
\mathcal{B}=\{\mathbf{m} \mid \mathbf{m} \text { satisfies DC and IC for } W(\Lambda)\}
$$

as a spanning set of $\mathbb{C}\left[\tilde{\Gamma}^{-}\right] / J_{\Lambda}$.

To see that $\overline{f_{\Lambda}}$ is an injection, note that $\overline{f_{\Lambda}}$ maps $\mathcal{B}$ bijectively onto

$$
\left\{\mathbf{m} v_{\Lambda} \mid \mathbf{m} \text { satisfies DC and IC for } W(\Lambda)\right\} \subset W(\Lambda),
$$

which is a basis of $W(\Lambda)$. This means that $\mathcal{B}$ is also linearly independent. Hence $\overline{f_{\Lambda}}$ maps a basis of $\mathbb{C}\left[\tilde{\Gamma}^{-}\right] / J_{\Lambda}$ onto a basis of $W(\Lambda)$ and therefore $\bar{f}_{\Lambda}$ is a bijection. 


\section{REFERENCES}

[1] E. Ardonne, R. Kedem and M. Stone, Fermionic characters and arbitrary highestweight integrable $\mathfrak{s} l_{r+1}$-modules, Comm. Math. Phys. 264 (2006), 427-464.

[2] I. Baranović, Combinatorial bases of Feigin-Stoyanovsky's type subspaces of level 2 standard modules for $D_{4}^{(1)}$, Comm. Algebra 39 (2011), 1007-1051.

[3] I. Baranović, M. Primc and G. Trupčević, Bases of Feigin-Stoyanovsky's type subspaces for $C_{\ell}^{(1)}$, Ramanujan J (2016). doi:10.1007/s11139-016-9840-y 1007-1051.

[4] M. Butorac, Combinatorial bases of principal subspaces for the affine Lie algebra of type $B_{2}^{(1)}$, J. Pure Appl. Algebra 218 (2014), 424-447.

[5] M. Butorac, Quasi particle bases of principal subspaces of the affine Lie algebra of type $G_{2}^{(1)}$, Glas. Mat. Ser. III 52(72) (2017), 79-98.

[6] C. Calinescu, Intertwining vertex operators and certain representations of $\widehat{\mathfrak{s l}(n)}$, Commun. Contemp. Math. 10 (2008), 47-79.

[7] C. Calinescu, J. Lepowsky and A. Milas, Vertex-algebraic structure of the principal subspaces of certain $A_{1}^{(1)}$-modules, I. Level one case, Internat. J. Math. 19 (2008), 71-92.

[8] C. Calinescu, J. Lepowsky and A. Milas, Vertex-algebraic structure of the principal subspaces of certain $A_{1}^{(1)}$-modules, II. Higher-level case, J. Pure Appl. Algebra 212 (2008), 1928-1950.

[9] C. Calinescu, J. Lepowsky and A. Milas, Vertex-algebraic structure of the principal subspaces of level one modules for the untwisted affine Lie algebras of types $A, D, E$, J. Algebra 323 (2010), 167-192.

[10] S. Capparelli, J. Lepowsky and A. Milas, The Rogers-Ramanujan recursion and intertwining operators, Commun. Contemp. Math. 5 (2003), 947-966.

[11] S. Capparelli, J. Lepowsky and A. Milas, The Rogers-Selberg recursions, the Gordon-Andrews identities and intertwining operators, Ramanujan J. 12 (2006), 379-397.

[12] A. V. Stoyanovsky and B. L. Feigin, Functional models of the representations of current algebras, and semi-infinite Schubert cells, (Russian) Funktsional. Anal. i Prilozhen. 28 (1994), 68-90; translation in Funct. Anal. Appl. 28 (1994), 55-72.

[13] B. Feigin, M. Jimbo, S. Loktev, T. Miwa and E. Mukhin, Bosonic formulas for $(k, \ell)$ admissible partitions, Ramanujan J. 7 (2003), 485-517.; Addendum to 'Bosonic formulas for $(k, \ell)$-admissible partitions', Ramanujan J. 7 (2003), 519-530.

[14] B. Feigin, M. Jimbo, T. Miwa, E. Mukhin and Y. Takeyama, Fermionic formulas for $(k, 3)$-admissible configurations, Publ. Res. Inst. Math. Sci. 40 (2004), 163-220.

[15] G. Georgiev, Combinatorial constructions of modules for infinite-dimensional Lie algebras. I. Principal subspace, J. Pure Appl. Algebra 112 (1996), 247-286.

[16] J. Humphreys Introduction to Lie algebras and representation theory, Springer, New-York, 1978.

[17] M. Jerković, Recurrence relations for characters of affine Lie algebra $A_{\ell}^{(1)}$, J. Pure Appl. Algebra 213, 913-926.

[18] M. Jerković and M. Primc, Quasi-particle fermionic formulas for $(k, 3)$-admissible configurations, Cent. Eur. J. Math. 10 (2012), 703-721.

[19] V. G. Kac, Infinite-dimensional Lie algebras, Cambridge University Press, Cambridge, 1990.

[20] J. Lepowsky and H.-S. Li, Introduction to vertex operator algebras and their representations, Birkhäuser, Boston, 2004.

[21] J. Lepowsky and M. Primc, Structure of the standard modules for the affine Lie algebra $A_{1}^{(1)}$, Contemporary Mathematics 46, American Mathematical Society, 1985. 
[22] A. Meurman and M. Primc, Annihilating fields of standard modules of $\mathfrak{s l}(2, \mathbb{C})^{\sim}$ and combinatorial identities, Mem. Amer. Math. Soc. 652, 1999.

[23] M. Penn, Lattice vertex superalgebras, I: Presentation of the principal subalgebra, Comm. Algebra 42 (2014), 933-961.

[24] M. Primc, Vertex operator construction of standard modules for $A_{n}^{(1)}$, Pacific J. Math. 162 (1994), 143-187.

[25] M. Primc, Basic representations for classical affine Lie algebras, J. Algebra 228 (2000), 1-50.

[26] M. Primc, Combinatorial bases of modules for affine Lie algebra $B_{2}^{(1)}$, Cent. Eur. J. Math. 11 (2013), 197-225.

[27] C. Sadowski, Presentations of the principal subspaces of the higher level standard $\widehat{s l(3)}$-modules, J. Pure Appl. Algebra 219 (2015), 2300-2345.

[28] A. V. Stoyanovsky, Lie algebra deformations and character formulas (Russian) Funktsional. Anal. i Prilozhen. 32 (1998), 84-86; translation in Funct. Anal. Appl. 32 (1998), 66-68.

[29] G. Trupčević, Bases of standard modules for affine Lie algebras of type $C_{\ell}^{(1)}$, Comm. Algebra 46 (2018), 3663-3673.

[30] G. Trupčević, Combinatorial bases of Feigin-Stoyanovsky's type subspaces of level 1 standard $\tilde{\mathfrak{s l}}(\ell+1, \mathbb{C})$-modules, Comm. Algebra 38 (2010), 3913-3940.

Faculty of Teacher Education

University of Zagreb

10000 Zagreb

Croatia

E-mail: goran.trupcevic@ufzg.hr

Received: 15.9.2017.

Revised: 20.11.2017. 